IHES/P/96/40

IASSNS-HEP-96/62

\title{
The Oklo bound on the time variation of the fine-structure constant revisited
}

\author{
Thibault Damour ${ }^{a, b, c}$, Freeman Dyson $^{b}$ \\ ${ }^{a}$ Institut des Hautes Etudes Scientifiques, \\ F-91440 Bures-sur-Yvette, France \\ ${ }^{b}$ School of Natural Sciences, Institute for Advanced Study, \\ Olden Lane, Princeton, NJ08540, U.S.A. \\ ${ }^{c}$ DARC, Observatoire de Paris-CNRS, \\ F-92195 Meudon, France.
}

June 27, 1996

\begin{abstract}
It has been pointed out by Shlyakhter that data from the natural fission reactors which operated about two billion years ago at Oklo (Gabon) had the potential of providing an extremely tight bound on the variability of the fine-structure constant $\alpha$. We revisit the derivation of such a bound by: (i) reanalyzing a large selection of published rare-earth data from Oklo, (ii) critically taking into account the very large uncertainty of the temperature at which the reactors operated, and (iii) connecting in a new way (using isotope shift measurements) the Oklo-derived constraint on a possible shift of thermal neutroncapture resonances with a bound on the time variation of $\alpha$. Our final (95\% C.L.) results are: $-0.9 \times 10^{-7}<\left(\alpha^{\text {Oklo }}-\alpha^{\text {now }}\right) / \alpha<1.2 \times 10^{-7}$ and $-6.7 \times 10^{-17} \mathrm{yr}^{-1}<\dot{\alpha}^{\text {averaged }} / \alpha<5.0 \times 10^{-17} \mathrm{yr}^{-1}$.
\end{abstract}




\section{Introduction}

Since Dirac [1] first suggested it as a possibility, the time variation of the fundamental constants has remained a subject of fascination which motivated numerous theoretical and experimental researches. For general discussions and references to the literature see, e.g., [2, 3, 4, 5]. Superstring theories have renewed the motivation for a variation of the "constants" by suggesting that most of the dimensionless coupling constants of physics, such as the fine structure constant $\alpha=1 / 137.0359895(61)$, are functions of the vacuum expectation values of some scalar fields (see, e.g., [6]). Recently, a mechanism for fixing the vacuum expectation values of such massless stringy scalar fields (dilaton or moduli) has been proposed [7]. This mechanism predicts that the time variation of the coupling constants, at the present cosmological epoch, should be much smaller than the Hubble time scale, but maybe not unmeasurably so. In this model, the time variations of all the coupling constants are correlated, and the ones of most observational significance are the fine structure constant $\alpha$ and the gravitational coupling constant $G$. In the present paper, we revisit the current best bounds on the variation of $\alpha$.

One of the early ideas for setting a bound on the variation of $\alpha$ was to consider the fine-structure splittings in astronomical spectra [8]. With this method, Bahcall and Schmidt [9] concluded that $\alpha$ had varied by at most a fraction $3 \times 10^{-3}$ of itself during the last $2 \times 10^{9}$ years. A recent update of this method has given the result $\triangle \alpha / \alpha=(0.2 \pm 0.7) \times 10^{-4}$ at redshifts $2.8 \leq z \leq$ 3.1, i.e. the bound $|\dot{\alpha} / \alpha|<1.6 \times 10^{-14} \mathrm{yr}^{-1}(2 \sigma$ level $)$ on the time derivative of $\alpha$ averaged over the last $\sim 10^{10} \mathrm{yr}$ [10. See also Ref. [11] which obtains $-4.6 \times 10^{-14} \mathrm{yr}^{-1}<\dot{\alpha} / \alpha<4.2 \times 10^{-14} \mathrm{yr}^{-1}$ from fine-structure splittings, and, denoting $x \equiv \alpha^{2} g_{p}\left(m_{e} / m_{p}\right),-2.2 \times 10^{-15} \mathrm{yr}^{-1}<\dot{x} / x<4.2 \times 10^{-15} \mathrm{yr}^{-1}$ by comparing redshifts obtained from hyperfine $(21 \mathrm{~cm})$ and optical data.

One of us obtained the upper limit $|\dot{\alpha} / \alpha|<5 \times 10^{-15} \mathrm{yr}^{-1}$ from an analysis of the abundance ratios of Rhenium and Osmium isotopes in iron meteorites and molybdenite ores [2]. The most recent direct laboratory test of the variation of $\alpha$ has obtained $|\dot{\alpha} / \alpha|<3.7 \times 10^{-14} \mathrm{yr}^{-1}$ by comparing hyperfine transitions in Hydrogen and Mercury atoms [12]. For more references on the variation of constants see [2, 3, 4, 5].

On the other hand, the much more stringent bound $|\dot{\alpha} / \alpha|<10^{-17} \mathrm{yr}^{-1}$ has been claimed by Shlyakhter [13, 14, 15] to be derivable (at the three standard deviations level) from an analysis of data from the Oklo phenomenon. 
The Oklo phenomenon denotes a natural fission reactor (moderated by water) that operated about two billion years ago in the ore body of the Oklo uranium mine in Gabon, West Africa. This phenomenon was discovered by the French Commissariat à l'Energie Atomique (CEA) in 1972. The results of a thorough, multi-disciplinary investigation of this phenomenon have been presented in two conference proceedings [16, [17]. See also [18] and [19] for summaries of the first phase of investigation.

In view of the importance of Shlyakhter's claim, of the lack of publication of a detailed analysis 叉, and of our dissatisfaction with some important aspects of the analysis presented in two preprints [14, 15], we decided to revisit the Oklo bound on $\alpha$. The main conclusions of our work are the following: (i) we confirm the basic claim of Shlyakhter that the Oklo data is an extremely sensitive probe of the time variation of $\alpha$; (ii) after taking into account various sources of uncertainty (notably temperature effects) in the analysis of data, and connecting in a improved way the raw results of this analysis to a possible variation of $\alpha$, we derive what we think is a secure (95\% C.L.) bound on the change of $\alpha$ :

$$
-0.9 \times 10^{-7}<\frac{\alpha^{\text {Oklo }}-\alpha^{\text {now }}}{\alpha}<1.2 \times 10^{-7} .
$$

In terms of an averaged rate of variation, this reads

$$
-6.7 \times 10^{-17} \mathrm{yr}^{-1}<\frac{\dot{\alpha}}{\alpha}<5.0 \times 10^{-17} \mathrm{yr}^{-1} .
$$

\section{Extracting the neutron capture cross sec- tion of Samarium 149 from Oklo data}

The proof of the past existence of a spontaneous chain reaction in the Oklo ore consists essentially of: (i) a substantial depletion of the Uranium isotopic ratio $1 \mathrm{U}^{235} / \mathrm{U}^{238}$ with respect to the current standard value in terrestrial samples; and (ii) a correlated peculiar distribution of some rare-earth isotopes. The rare-earth isotopes are abundantly produced in the fission of

\footnotetext{
${ }^{1}$ The very brief account published in Nature [13] omits most of the analysis that is presented in the two preprints [14, 15].

${ }^{2}$ For typographical convenience, we indicate atomic mass numbers as right, rather than left, superscripts.
} 
$\mathrm{U}^{235}$ and the observed isotopic distribution is beautifully consistent with calculations of the effect of a strong neutron flux on the fission yields of $U^{235}$ (see e.g. 20, 21, 22, 23, 24, 251). In particular, the strong neutron absorbers $\mathrm{Sm}^{149}, \mathrm{Eu}^{151}, \mathrm{Gd}^{155}$ and $\mathrm{Gd}^{157}$ are found in very small quantities in the central regions of the Oklo reactors (see, e.g., Fig. 2 of [23]). These isotopes were evidently burned up by the large neutron fluence produced by the fission process. Following Shlyakhter's suggestion [14, 15], we concentrate on the determination of the neutron capture cross section of $\operatorname{Sm}^{149}: \operatorname{Sm}^{149}(n, \gamma)$ $\mathrm{Sm}^{150}$.

The evolution of the concentrations of the various Samarium isotopes (sharing the common atomic number $Z=62$ ) in the Oklo ore is especially simple to describe because of the absence of a stable chemical element with atomic number $Z=61$. The most stable Promethium nuclide is $\operatorname{Pm}_{61}^{145}$ with a half-life of 17.7 years. If there had existed, before the reaction started, some natural concentration of Promethium it could, via neutron absorption and subsequent $\beta^{-}$decay, have generated some Samarium. In absence of this, the final values of the Sm concentrations are determined by: (i) their initial concentrations, before the reaction; (ii) the yields from the fissions; and (iii) the effect of neutron captures.

Following Refs. [16, 17], one characterizes the neutron absorbing power of an isotope by the effective cross section

$$
\hat{\sigma} \equiv \frac{\int \sigma(E) v n_{E} d E}{v_{0} \int n_{E} d E}
$$

where $v$ is the (relative) velocity of incident neutrons, $n_{E} d E$ the energy distribution of the neutrons, and $v_{0}$ the fiducial (thermal) velocity $v_{0}=2200 \mathrm{~m} / \mathrm{s}$ corresponding to a kinetic energy $E_{0}=0.0253 \mathrm{eV}$. The advantage of the definition (3) is that, in the case of a " $1 / v$ absorber", $\sigma(E)=C / v$, the effective cross section equals $\hat{\sigma}=C / v_{0}=\sigma\left(E_{0}\right)$ independently of the neutron spectrum. [To a good appoximation, this is the case for the thermal fission cross section of $\left.\mathrm{U}^{235}\right]$. On the other hand, in the case of nuclides exhibiting resonances in the thermal region (these are the strong absorbers, $\mathrm{Sm}^{149}, \mathrm{Eu}^{151}$, $\mathrm{Gd}^{155}, \mathrm{Gd}^{157}$ ), the value of the effective cross section (3) is very sensitive to the neutron spectrum, especially to its thermal part p.

\footnotetext{
3 The spectrum of moderated neutrons in a fission reactor consists of a MaxwellBoltzmann thermal distribution up to energies of order a few times $k T$, followed by a tail $n_{E} d E \propto d E /(v E)$ due to neutrons still in the process of moderation
} 
Associated to the introduction of the effective cross section (3), one defines an effective neutron flux $\hat{\phi} \equiv n v_{0}$ with $n=\int n_{E} d E$, and an effective infinitesimal fluence (integrated flux)

$$
d \tau=\hat{\phi} d t=n v_{0} d t
$$

With this notation, the general equation describing the evolution of the total number $N_{A}$ of nuclides of mass number $A$ (for some fixed atomic number $Z$ ) in some sample reads

$$
\frac{d N_{A}}{d \tau}=y_{A} N_{5} \sigma_{f 5}+\sigma_{A-1} N_{A-1}-\sigma_{A} N_{A}
$$

Here, $y_{A}$ denotes the yield of the element $A$ in the fission of $\mathrm{U}^{235}, N_{5}$ and $\sigma_{f 5}$ are short hands for $N_{235}$ and the (effective) fission cross section of $\mathrm{U}^{235}$, and the last two terms describe the effects of neutron captures within isotopes of some chemical element $Z$. For simplicity, we drop the carets over the cross sections. The evolution equation (5) neglects any contribution $\propto N_{A-1}\left(Z^{\prime}\right)$ coming from the $\beta^{-}$decay of the neighbouring chemical element $Z^{\prime}=Z-1$ after absorption of a neutron. As we said above, this approximation applies well to the Samarium case. Eq. (5) neglects also the yields due to the fractionally small number of fissions of $\mathrm{U}^{238}$ and $\mathrm{Pu}^{239}$. [See, e.g., Ref. [23] which estimates that, in a particular sample, $2.5 \%$ and $3 \%$ of the fissions were due to $\mathrm{U}^{238}$ and $\mathrm{Pu}^{239}$, respectively].

Samples in the cores of the various Oklo reactors were exposed to a total effective fluence $\tau=\int d \tau=\int n v_{0} d t$ of the order of $10^{21}$ neutron $/ \mathrm{cm}^{2}=1$ inverse kilobarn. This means, roughly speaking, that processes with effective cross sections comparable or larger than $1 \mathrm{~kb}$ have led to a significant number of reactions, while processes with $\sigma \ll 1 \mathrm{~kb}$ had a negligible effect. The former category includes the fission of $\mathrm{U}^{235}\left(\sigma_{f 5} \sim 0.6 \mathrm{~kb}\right)$, the capture of neutrons by $\operatorname{Nd}^{143}\left(\sigma_{143} \sim 0.3 \mathrm{~kb}\right)$ and by the strong absorbers (such as $\left.\mathrm{Sm}^{149} ; \sigma_{149} \gtrsim 70 \mathrm{~kb}\right)$, while the latter category includes neutron captures by weak absorbers such as $\mathrm{Sm}^{144}$ and $\mathrm{Sm}^{148}$ with cross sections of only a few barns.

This allows one to neglect $\sigma_{144}$ and $\sigma_{148}$ (for $Z=62$ ) in Eq. (5). Further simplification comes from the fact that the stable isotopes 144, 146 and 148 of Neodymium prevent the formation of the long-livedf $\mathrm{Sm}^{144}, \mathrm{Sm}^{146}$ and

\footnotetext{
${ }^{4}$ The half-life of $\mathrm{Sm}^{146}$ is $1.03 \times 10^{8} \mathrm{yr}$ and therefore long with respect to the duration of the Oklo phenomenon.
} 
$\mathrm{Sm}^{148}$ as end points of ( $\beta^{-}$decay) fission chains. The stable isotopesf obey the simple evolution equations

$$
\begin{aligned}
\frac{d N_{144}}{d \tau} & =0, \\
\frac{d N_{147}}{d \tau} & =y_{147} N_{5} \sigma_{f 5}-\sigma_{147} N_{147}, \\
\frac{d N_{148}}{d \tau} & =\sigma_{147} N_{147}, \\
\frac{d N_{149}}{d \tau} & =y_{149} N_{5} \sigma_{f 5}-\sigma_{149} N_{149}, \\
\frac{d N_{5}}{d \tau} & =-N_{5} \sigma_{5}^{*} .
\end{aligned}
$$

To close the system, we have followed [22 and [23] in describing the burn up of $\mathrm{U}^{235}$ by means of a modified absorption cross section $\sigma_{5}^{*}=\sigma_{5}(1-C)$, where $\sigma_{5}$ is the normal absorption cross section (fission plus capture) and $C$ is a conversion factor representing the formation of $\mathrm{U}^{235}$ from the decay of $\mathrm{Pu}^{239}$ formed by neutron capture in $\mathrm{U}^{238}$.

In the approximation where the (effective) cross sections, and the conversion factor $C$, are constant, the system (6-10) is easily solved and gives

$$
\begin{aligned}
N_{5}(\tau) & =N_{5}(0) e^{-\sigma_{5}^{*} \tau} \\
N_{144}(\tau) & =N_{144}(0) \\
N_{147}(\tau)+N_{148}(\tau) & =N_{147}(0)+N_{148}(0)+y_{147} \sigma_{f 5} N_{5}(0) \frac{1-e^{-\sigma_{5}^{*} \tau}}{\sigma_{5}^{*}} \\
N_{149}(\tau) & =N_{149}(0) e^{-\sigma_{149} \tau}+y_{149} \sigma_{f 5} N_{5}(0) \frac{e^{-\sigma_{5}^{*} \tau}-e^{-\sigma_{149} \tau}}{\sigma_{149}-\sigma_{5}^{*}}
\end{aligned}
$$

Eq. (12) shows that the quantity of $\mathrm{Sm}^{144}$ measured in a sample now is equal to the quantity of natural $\mathrm{Sm}^{144}$ present in the sample before the nuclear reactions. Assuming that the natural Samarium present in the sample at the beginning had the normal isotopic ratios ( say $n_{144}=3.1 \%, n_{147}=15.0 \%$, $n_{148}=11.3 \%, n_{149}=13.8 \%$, etc... [26]), we can use (12] to correct (13]) for the initial concentrations in $\mathrm{Sm}^{147}$ and $\mathrm{Sm}^{148}$. The effect of $N_{149}(0)$

\footnotetext{
5 "Stable" means, in this context, a half life much larger than the age of the Oklo phenomenon. E.g. the half-life of $\mathrm{Sm}^{147}$ is $1.06 \times 10^{11} \mathrm{yr} \gg 2 \times 10^{9} \mathrm{yr}$.
} 
in Eq. (14) is totally negligible (as is the last term) because the exponent $\sigma_{149} \tau \gg 1$. We then derive the intermediate result

$$
\frac{N_{147}(\tau)+N_{148}(\tau)-\frac{n_{147}+n_{148}}{n_{144}} N_{144}(\tau)}{N_{149}(\tau)}=\frac{y_{147}}{y_{149}} \frac{e^{\sigma_{5}^{*} \tau}-1}{\sigma_{5}^{*}}\left(\sigma_{149}-\sigma_{5}^{*}\right) .
$$

One can finally obtain an expression for $\sigma_{149}$ in terms of "measured" quantities by connecting $\sigma_{5}^{*} \tau$ to the observed ratio between the numbers of $\mathrm{U}^{235}$ and $\mathrm{U}^{238}$ atoms in the Oklo sample. If, following [22], we define

$$
w \equiv \frac{0.00725}{\left(N_{5} / N_{8}\right)_{\text {now }}^{\text {Oklo }}},
$$

where 0.00725 is the usual $\mathrm{U}^{235} / \mathrm{U}^{238}$ ratio in natural Uranium now, it is easy to verify that $w=e^{\sigma_{5}^{*} \tau}$. Finally, we get

$$
\sigma_{149}=\frac{1}{\tau}\left[\ln w+y \frac{\ln w}{w-1} \frac{N_{147}+N_{148}-n N_{144}}{N_{149}}\right],
$$

where (using Refs. [27] and [26])

$$
\begin{aligned}
& y=\frac{y_{149}}{y_{147}}=\frac{1.080384}{2.261681} \simeq 0.478, \\
& n=\frac{n_{147}+n_{148}}{n_{144}}=\frac{15.0+11.3}{3.1} \simeq 8.48 .
\end{aligned}
$$

The quantities $N_{A}$ in Eq. (17) denote the present values of the isotopic concentrations, or, equivalently, the present values of the isotopic ratios. The isotopic ratios of Samarium have been measured in many Oklo samples 28, 20, 23, 29, 24]. Note that the quantity which is, at this stage, directly obtainable from observations is the dimensionless product $\sigma_{149} \tau=$ $\int d t \int \sigma_{149}(E) v n_{E} d E$.

Similarly, by considering the fission yields of Neodymium and the neutroncapture reaction $\mathrm{Nd}^{143} \rightarrow \mathrm{Nd}^{144}$ (using, e.g., the $\mathrm{Nd}^{142}$ content to subtract the contribution from the natural concentrations present before the reaction),

\footnotetext{
${ }^{6} \mathrm{Eq} .(17)$ is equivalent to equations appearing in Refs. [22, 14, 15] apart from the facts that Shlyakhter's equations contain misprints (e.g. $(w-1) / \ln w$ instead of its inverse in Eq. (17)). The fractionally small first contribution on the right-hand side of (17) is neglected in the above references.
} 
several authors [20, 21, 22] have shown how to obtain the dimensionless product $\sigma_{143} \tau$ (where $\sigma_{143} \equiv \sigma_{(n, \gamma)}\left(\mathrm{Nd}^{143}\right)$ ) in terms of quantities observed in Oklo samples. Combining these two results, we see that the value two billion years ago of the ratio $\sigma_{149} \tau / \sigma_{143} \tau=\sigma_{149} / \sigma_{143}$ can be computed in terms of present Oklo data.

Although it would be conceptually clearer to deal only with the dimensionless ratio $\sigma_{149} / \sigma_{143}$, we shall follow previous usage in working with the dimensionful quantity $\sigma_{149}$ obtained by inserting in Eq. (17) the value of the (effective) fluence $\tau$ deduced from Neodymium data by previous authors. This procedure is justified by the fact that the effective cross-section $\sigma_{143}$, defined by Eq. (3), depends very little on the neutron spectrum because $\sigma_{143}(E)$ follows the $1 / v$ law over most of the range of interest. Therefore the lack of knowledge of the temperature of the moderated neutrons is of no importance (contrary to the case of $\sigma_{149}$ ) and the effect of epithermal neutrons is also very small'. In other words, the extraction of $\sigma_{143} \tau$ from Oklo data is approximately done by assuming a fixed, fiducial value for $\sigma_{143}$, say $\sigma_{143} \simeq 325 \mathrm{~b}$, so that the use of Eq. (17) for computing a dimensionful $\sigma_{149}$ is approximately equivalent to computing the dimensionless quantity $325 \sigma_{149} / \sigma_{143}$.

The detailed isotopic analysis of Oklo data [16, 17] has shown that, generally speaking, the ore composition has changed very little since the end of the nuclear reactions. This is established by studying the correlation between the fluence $\tau$ and the Uranium isotopic ratio $N_{5} / N_{8}$, and by showing that it can be explained by neutronics considerations (see, e.g. [29]). However, in some cases there is evidence for a partial reshuffling of chemical elements after the end of the reactions. We have examined these results and selected 16 samples as especially suitable for extracting a reliable value of $\sigma_{149}$. These samples are all core samples with high Uranium content, large depletions of $\mathrm{U}^{235}$, and high fluences, $\tau \gtrsim 0.7 \times 10^{21} \mathrm{n} / \mathrm{cm}^{2}$. In all cases, the natural element correction in Eq. (17) is small, the observed Samarium having been produced almost entirely by fission. The very small content of $\mathrm{Sm}^{149}$ (and, when data are available, of other strong absorbers such as $\mathrm{Gd}^{155}$ and $\mathrm{Gd}^{157}$ ) is also a confirmation of the absence of chemical reshuffling after the reaction. The data we took come from [28, 22, 23, 29, 24], and [31]

\footnotetext{
${ }^{7}$ In the analysis of Oklo data, it has been customary to parametrize the contribution of epithermal neutrons to the spectrum by a parameter called $r$. This parameter is found to be small, $r \sim 0.15$, and its effect on $\sigma_{143}$ is only a few percent 30.

${ }^{8}$ The sample SC521472 taken from this last reference was exposed to a smaller fluence
} 
calculating $\sigma_{149}$ from these data is exhibited in Table I.

The large scatter of the values exhibited in Table I is compatible with the strong temperature dependence of $\sigma_{149}$ (see below). The only exception is the $36 \mathrm{~kb}$ obtained for the sample SC39-1387. This value is a clear outlier which, most plausibly, has been contaminated in some way. Excluding this result, the other 15 results are all contained in the range

$$
57 \mathrm{~kb} \leq \hat{\sigma}_{149} \leq 93 \mathrm{~kb} .
$$

We think that it is conservative to consider the full range $(20)$ as a " $2 \sigma$ " (or $95 \%$ C.L.) interval for $\hat{\sigma}_{149}$. [For clarity, we reestablish the caret meaning that we are dealing with an effective cross section, Eq. (3).] Actually, in view of what is known from Oklo, it is very plausible that the range (20) is to be attributed to a mixture of temperature effects and a small amount of post-reaction chemical reshuffling. For our purpose, we will use the full range (20) to define a conservative bound on the variation of $\alpha$. For completeness, and in view of the special use we make of the interval (20) we give in Table II the complete set of data allowing one (using (17)) to compute $\hat{\sigma}_{149}$ for the samples giving the extreme values (20).

Let us note that the values we obtain for $\hat{\sigma}_{149}$ are different from the result claimed by Shlyakhter [14, 15], namely $\hat{\sigma}_{149}=(55 \pm 8) \mathrm{kb}$. As he did not mention the data he used, we could not trace the origin of this difference. We note that most of the values in Table I are compatible with thermal effects $\left(\hat{\sigma}_{149}\right.$ increases from $\sim 70 \mathrm{~kb}$ to $\sim 99 \mathrm{~kb}$ when the temperature varies

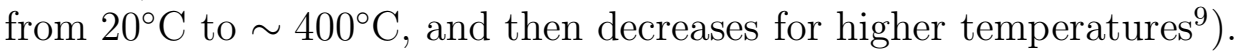

\section{Bounding a possible shift of the lowest res- onance in the capture cross section of Samar- ium 149}

Following Shlyakhter's suggestion [13, 14, 15], we shall translate the range of "Oklo" values of $\hat{\sigma}_{149}$, Eq. (20), into a bound on the possible shift, between

than the others. It was included because this sample has been used to estimate the temperature of the neutrons.

${ }^{9}$ The fact that we did not find values between 93 and $99 \mathrm{~kb}$ is probably explained by some post-reaction remobilization. Anyway, the limit we shall derive on $\alpha$ depends only on the lower bound on $\hat{\sigma}_{149}$. 
the time of the Oklo phenomenon and now, of the lowest resonance in the monoenergetic cross section $\sigma_{149}(E)$. The large values of the thermal capture cross sections of $\mathrm{Sm}^{149}, \mathrm{Gd}^{155}$ and $\mathrm{Gd}^{157}$ are due to the existence of resonances in the thermal region. In presence of such a resonance, the monoenergetic capture cross section is well described, in the thermal region, by the BreitWigner formula

$$
\sigma_{(n, \gamma)}(E)=\pi \frac{\hbar^{2}}{p^{2}} g \frac{\Gamma_{n}(E) \Gamma_{\gamma}}{\left(E-E_{r}\right)^{2}+\frac{1}{4} \Gamma^{2}} .
$$

Here $p$ is the momentum of the neutron, $E=p^{2} / 2 m_{n}$ its kinetic energy, $g=\left(2 I^{\prime}+1\right)(2 s+1)^{-1}(2 I+1)^{-1}$ a statistical factor depending upon the spins of the compound nucleus $I^{\prime}$, of the incident neutron $s=\frac{1}{2}$, and of the target nucleus $I, \Gamma_{n}(E)$ is a neutron partial width, $\Gamma_{\gamma}$ a radiative partial width, and $\Gamma$ the total width. The neutron partial width $\Gamma_{n}(E)$ varies approximately as $E^{1 / 2}$

$$
\Gamma_{n}(E)=\frac{2 \gamma_{n}^{2}}{\hbar} p
$$

where $\gamma_{n}^{2}$ is a "reduced partial width" (see, e.g., [32]). With sufficient approximation, the total width is given by

$$
\Gamma \simeq \Gamma_{\gamma}+\Gamma_{n}\left(E_{r}\right)
$$

As we shall see explicitly below, the position of the resonance $E_{r}$ (with respect to the threshold defined by zero-kinetic-energy incident neutrons) is extremely sensitive to the value of the fine-structure constant. By contrast, the other quantities entering the Breit-Wigner formula, $\gamma_{n}^{2}, \Gamma_{\gamma}$, have only a mild (polynomial) dependence on $\alpha$. Therefore the sensitivity to $\alpha$ of the effective cross sections $\hat{\sigma}$, Eq. (3), measured with Oklo data is totally dominated, for strong absorbers, by the dependence of $\sigma(E)$ upon the position of the lowest lying resonance $E_{r}$. As we said above, we should, more rigourously, work with dimensionless ratios such as $\hat{\sigma}_{149} / \hat{\sigma}_{143}$. However, the mild absorber $\mathrm{Nd}^{143}$ has no resonances in the thermal region. Therefore the $\alpha$-sensitivity of the ratio $\hat{\sigma}_{149} / \hat{\sigma}_{143}$ is completely dominated by the $\alpha$-sensitivity of $\hat{\sigma}_{149}$ inherited from the dependence on $E_{r}^{149}(\alpha)$.

The main problem is to use the range $(20)$ of values of $\hat{\sigma}_{149}$ to put a limit on a possible shift of the lowest lying resonance in $\mathrm{Sm}^{149}$,

$$
\Delta \equiv E_{r}^{149(\text { Oklo) }}-E_{r}^{149 \text { (now) }} .
$$


Previous attempts [13, 14, 15, 4] at relating Oklo-deduced ranges of values of $\hat{\sigma}_{149}$ to $\Delta$ are unsatisfactory because they did not properly take into account the very large uncertainty in $\hat{\sigma}_{149}$ due to poor knowledge of the neutron temperature in the Oklo reactors. The original analysis of Shlyakhter assumed a temperature $T \simeq 20^{\circ} \mathrm{C}$ (which is much too low), and the analysis of [ took $T \simeq 1000 \mathrm{~K}$, i.e. $T \simeq 725^{\circ} \mathrm{C}$ (which is possible, but on the high side) and assumed that one could work linearly in the fractional shift $\Delta / E_{r}$. We think that one should neither fix the neutron temperature $T$ (which could have varied over a wide range), nor work linearly in $\Delta / E_{r}$ (which could have been larger than unity).

Several studies, using independent data, have tried to constrain the value of the temperature in the Oklo reactors [17]. Mineral phase assemblages observed within a few meters of the Oklo reactor zones 2 and 5 indicate a minimum temperature in these regions of about $400^{\circ} \mathrm{C}$, while relict textures in the reactor zone rock suggest that temperatures $T \simeq 650-700^{\circ} \mathrm{C}$ may have been reached within the reactors [33]. A study of fluid inclusions and petrography of the sandstones suggest pressures $p \simeq 800-1000$ bar $\square$ and temperatures ranging between $180^{\circ} \mathrm{C}$ and at least $600^{\circ} \mathrm{C}$. On the other hand, the temperature of the water-moderated neutrons during the fission reactions has been evaluated by a study of the $\mathrm{Lu}^{176} / \mathrm{Lu}^{175}$ and $\mathrm{Gd}^{156} / \mathrm{Gd}^{155}$ isotope ratios in several samples. The values obtained range between $250 \pm 40^{\circ} \mathrm{C}$ and $450 \pm 20^{\circ} \mathrm{C}$ depending upon the sample and the isotope ratio considered [31]. It is to be noted that the concentration of strong absorbers such as $\mathrm{Sm}^{149}$ or $\mathrm{Gd}^{155}$ (which are burned very efficiently) is determined by the values of the effective cross sections $\hat{\sigma}_{149}$ or $\hat{\sigma}_{155}$ at the end of the fission phenomenon. Therefore, we cannot exclude that the temperature to be used in evaluating $\hat{\sigma}_{149}$ or $\hat{\sigma}_{155}$ be on the low side of the allowed range.

Summarizing, we consider that the temperature to be used to determine $\hat{\sigma}_{149}$ or $\hat{\sigma}_{155}$ could be in the full range

$$
180^{\circ} \mathrm{C} \leq T \lesssim 700^{\circ} \mathrm{C} .
$$

\footnotetext{
${ }^{10}$ It is thought that the Oklo phenomenon took place while the Uranium deposits were buried $\sim 4 \mathrm{~km}$ deep [34].
} 
Inserting a Maxwell-Boltzmann spectrum th

$$
\frac{n_{E}}{n} d E=\frac{2 \pi}{(\pi k T)^{3 / 2}} e^{-\frac{E}{k T}} E^{1 / 2} d E
$$

in the definition (3), with $\sigma(E)$ of the Breit-Wigner form (21), we find that the dependence of the effective cross section of a strong absorber on resonance shift $\Delta$, Eq. (24), and temperature is given by

$$
\hat{\sigma}(\Delta, T)=\frac{2 \pi}{(\pi k T)^{3 / 2}} \sigma_{0}\left(1+y_{0}^{2}\right) \int_{0}^{\infty} \frac{e^{-\frac{E}{k T}} E^{1 / 2} d E}{1+y^{2}(E, \Delta)} .
$$

Here $\sigma_{0}$ denotes the "thermal" radiative cross section (as observed now), i.e. the monoenergetic $\sigma_{(n, \gamma)}\left(E_{0}\right)$ evaluated at $E_{0} \equiv 0.0253 \mathrm{eV}, y_{0}$ denotes $2\left(E_{0}-E_{r}^{\text {now }}\right) / \Gamma$, and $y(E, \Delta) \equiv 2\left(E-E_{r}^{\text {now }}-\Delta\right) / \Gamma$. The $E^{1 / 2}$ in the integrand comes from combining several different factors: a factor $E^{-1 / 2}$ coming from $\Gamma_{n}(E) / p^{2}\left(1 / v\right.$ law), a factor $E^{1 / 2}$ coming from the factor $v$ in Eq. (3), and the factor $E^{1 / 2}$ in the Maxwell spectrum (26).

In the case of $\mathrm{Sm}^{149}$ the numerical values needed to evaluate (27) are (from 35]): $E_{r}^{\text {now }}=0.0973 \mathrm{eV}, \sigma_{0}=40.14 \mathrm{~kb}$, and $\Gamma \simeq 0.061 \mathrm{eV}$. [The latter being estimated from $\Gamma \simeq \Gamma_{\gamma}+\Gamma_{n}\left(E_{r}\right)$ with $\Gamma_{\gamma}=60.5 \times 10^{-3} \mathrm{eV}$, $2 g \Gamma_{n}\left(E_{r}\right)=0.6 \times 10^{-3} \mathrm{eV}$, with $2 g=9 / 8$ corresponding to $I^{\prime}=4$ and $I=7 / 2$.] The dependence of $\hat{\sigma}_{149}$ upon the resonance shift $\Delta$ is shown in Fig. 1 for several temperatures spanning the range (25). On the same Figure, we have indicated the conservative range of values of $\hat{\sigma}_{149}$, Eq. (20).

The limits on $\Delta$ shown in Fig. 1 are $-0.12 \mathrm{eV}<\Delta<0.08 \mathrm{eV}$. The lower limit depends on the minimal allowed temperature. Given the temperature estimates quoted above, we consider $180^{\circ} \mathrm{C}$ as a firm minimal temperature and therefore $-0.12 \mathrm{eV}$ as a firm lower bound. As the upper limit $0.08 \mathrm{eV}$ depends on the maximum allowed temperature which is more uncertain, we have also explored temperatures higher than $700^{\circ} \mathrm{C}$. We found that when $\Delta=0.09 \mathrm{eV} \hat{\sigma}$ never exceeds $57 \mathrm{~kb}$ even if $T$ is allowed to take values much larger than $700^{\circ} \mathrm{C}$. $[\hat{\sigma}(0.09, T)$ reaches a maximum $<57 \mathrm{~kb}$ somewhere around $T \sim 1000^{\circ} \mathrm{C}$.] Therefore to be conservative, we take $\Delta<0.09 \mathrm{eV}$ as firm

\footnotetext{
${ }^{11}$ We do not consider the effect of epithermal neutrons, which introduce only a rather small fractional correction (spectrum index of order $r \sim 0.15$ [16, 17]). This correction is negligible compared to the wide range we consider.
} 
upper limit. We conclude that the Oklo Samarium data constrain a possible resonance shift to be in the range

$$
-0.12 \mathrm{eV}<\Delta<0.09 \mathrm{eV}
$$

For comparison, let us mention that Refs [13, 14, 15] estimate a $2 \sigma$ range $|\Delta|<0.02 \mathrm{eV}$ from the Samarium data alone, and a $3 \sigma$ range $|\Delta|<0.05 \mathrm{eV}$ from combining Samarium and Europium data.

We tried to make use of Oklo Gadolinium data to restrict further the Samarium-derived range (28). A priori, one could think of making use of both $\mathrm{Gd}^{155}$ and $\mathrm{Gd}^{157}$ which are strong absorbers of neutrons. In fact, $\mathrm{Gd}^{157}$ is such a strong absorber that its final concentration $N_{157} \propto y_{157} / \hat{\sigma}_{157}$ (generalizing Eq. (14)) is too small to be measured reliably. The case of $\mathrm{Gd}^{155}$ is more favorable, its effective cross section being comparable to that of $\mathrm{Sm}^{149}$. However, its fission yield is much smaller $\left(y_{155}=0.032 \%\right.$ instead of $y_{149}=1.08 \%$ ). The absolute concentration of all isotopes of Gadolinium is about ten times smaller than that of Samarium (see, e.g., 23]). This implies that Gadolinium data are much more prone to various contaminations (natural element contamination due to a post-reaction remobilization, and uncertainties in the isotopic analysis measurements). To make a meaningful analysis of Gadolinium data, one should probably restrict oneself to samples that were exposed to rather mild fluences. Such samples are SC361901 and SC521472 which have been studied in detail in [31]. The effective cross sections $\hat{\sigma}\left(\mathrm{Gd}^{155}\right)$ obtained in the latter reference are $\hat{\sigma}_{155}=(42.0 \pm 0.5) \mathrm{kb}$ in SC 361901, and $\hat{\sigma}_{155}=(32.5 \pm 0.5) \mathrm{kb}$ in SC 521472. These values are compatible with the present values of $\hat{\sigma}_{155}$ if the temperatures in these samples were $T_{361901} \simeq 380^{\circ} \mathrm{C}$ and $T_{521472} \simeq 450^{\circ} \mathrm{C}$. [Actually, these temperatures disagree with the Lutetium-derived ones: $T_{361901}^{\mathrm{Lu}} \simeq 250^{\circ} \mathrm{C}$ and $T_{521472}^{\mathrm{Lu}} \simeq 280^{\circ} \mathrm{C}$. This difference is probably to be explained by a moderate amount of contamination of natural Lutetium after the reaction [31.] However, we could not use these data to derive more stringent limits on $\alpha$ because the $\mathrm{Gd}^{155}$ resonance turns out to be less than half as sensitive as Samarium to changes in $\alpha$ (see next Section). 


\section{Translating possible resonance shifts into a bound on the variation of the fine-structure constant.}

Let us finally translate the allowed range (28) into a bound on a possible difference between the value of $\alpha$ during the Oklo phenomenon and its value now. The treatments given in previous analyses are unsatisfactory. The original analysis of Shlyakhter [13, 14, 15] rested on a coarse representation of the nucleus as a square potential well, together with dubious assumptions about nuclear compressibility, while the analysis of Ref. [⿴囗十 used an ill-motivated finite-temperature description of the excited state of the compound nucleus.

The observed neutron-resonance energy $E_{r}^{\text {now }}=0.0973 \mathrm{eV}$, for the radiative capture of neutrons by $\mathrm{Sm}_{62}^{149}$, corresponds to the existence of a particular excited quantum state of $\mathrm{Sm}_{62}^{150}$. More precisely, if we write the total massenergy of the relevant excited state of $\mathrm{Sm}_{62}^{150}$ as $\llbracket E_{150}^{*}=62 m_{p}+88 m_{n}+E_{1}$ (with $E_{1}<0$ ), and the total mass-energy of the ground state of $\operatorname{Sm}_{62}^{149}$ as $E_{149}=62 m_{p}+87 m_{n}+E_{2}$ (with $E_{2}<0$ ), we have

$$
E_{r}=E_{150}^{*}-E_{149}-m_{n}=E_{1}-E_{2} .
$$

Both $E_{1}$ and $E_{2}$ are eigenvalues of the Hamiltonian

$$
H=H_{n}+H_{c}
$$

where $H_{c}$ is the Coulomb energy

$$
H_{c}=e^{2} \sum R_{i j}^{-1}
$$

summed over the pairs of protons in the nucleus, and $H_{n}$ is, to a good accuracy, independent of $e^{2}$. We neglect small effects such as the magneticmoment interactions or the QED corrections to the masses, such as $m_{n}$ and $m_{p}$, entering the nuclear Hamiltonian $H_{n}$.

Now let $e^{2}$ vary while $H_{n}$ remains fixed. Then for any eigenstate of $H$ with eigenvalue $E$,

$$
e^{2} d E / d e^{2}=\left\langle H_{c}\right\rangle
$$

\footnotetext{
${ }^{12}$ We set $c=1$.
} 
and therefore

$$
e^{2} d E_{r} / d e^{2}=\left\langle H_{c}\right\rangle_{1}-\left\langle H_{c}\right\rangle_{2} .
$$

The Coulomb energies on the right of (33) are not directly measurable. The quantities that can be directly measured by optical spectroscopy [36] are the mean-square radii $\left\langle r^{2}\right\rangle$ of the charge-distributions of the protons in the various isotopes of Samarium. Let us recall that "isotope shifts" in heavy atoms are related to the effect of the finite extension of the nucleus on electron energies. A first-order perturbation analysis of the latter effect (see, e.g., 37]) yields $\Delta E=(2 \pi / 3) \psi_{e}^{2}(0) Z e^{2}\left\langle r^{2}\right\rangle$ where $\psi_{e}(r)$ is an (s-state) electron wave function, and where $\left\langle r^{2}\right\rangle=Z^{-1} \int \rho r^{2} d v$ with $\rho$ denoting the proton charge distribution in the nucleus.

To connect the expectation-values in (33) with the mean-square radii, we use the semi-classical approximation

$$
\left\langle H_{c}\right\rangle_{i}=\frac{1}{2} e^{2} \int V_{i} \rho_{i} d v, i=1,2,
$$

where $\rho_{i}$ is the density of protons in the nuclear state $i$, normalized to

$$
\int \rho_{i} d v=Z=62
$$

for Samarium, and $V_{i}$ is the electrostatic potential generated by $\rho_{i}$. From (33) and (34),

$d E_{r} / d e^{2}=\frac{1}{2} \int\left(V_{1} \rho_{1}-V_{2} \rho_{2}\right) d v=-\left(\frac{1}{2}\right) \int \delta V \delta \rho d v+\int V_{1} \delta \rho d v<\int V_{1} \delta \rho d v$,

with

$$
\delta \rho=\rho_{1}-\rho_{2}, \quad \delta V=V_{1}-V_{2} .
$$

The term that is dropped in (36) is negative because it is minus an electrostatic self-energy. The integrand on the right side of (36) is a small difference $\delta \rho$ multiplied by the smooth potential $V_{1}$. With an error of second order in small quantities, we may approximate $V_{1}$ by the classical potential of a uniformly charged sphere with radius $R_{1}$,

$$
V_{1}(r)=Z\left[\frac{3 R_{1}^{2}-r^{2}}{2 R_{1}^{3}}\right] .
$$


Then (36) becomes

$$
\frac{d E_{r}}{d e^{2}}<-\left[\frac{Z^{2}}{2 R_{1}^{3}}\right] \delta_{12}\left(r^{2}\right),
$$

where

$$
\delta_{12}\left(r^{2}\right)=\frac{1}{Z} \int r^{2} \delta \rho d v
$$

is the difference in mean-square charge-radius between the states 1 and 2 .

Let the label 3 denote the ground-state of $\mathrm{Sm}^{150}$. Then

$$
\delta_{12}\left(r^{2}\right)=\delta_{13}\left(r^{2}\right)+\delta_{32}\left(r^{2}\right) .
$$

The difference $\delta_{13}\left(r^{2}\right)$ cannot be calculated because we do not know the shape of the excited state of $\mathrm{Sm}^{150}$. But it seems safe to assume that the proton charge distribution will not be more tightly concentrated in the excited state than in the ground state. That is to say,

$$
\delta_{13}\left(r^{2}\right) \geq 0 \text {. }
$$

An inequality stronger than (42) could be deduced from more dubious assumptions about nuclear compressibility, but a stronger inequality is not needed. From (39) and (42) we have

$$
\frac{d E_{r}}{d e^{2}}<-\left[\frac{Z^{2}}{2 R_{1}^{3}}\right] \delta_{32}\left(r^{2}\right),
$$

and this is sufficient for our purposes. The experimental isotope-shift measurements reported by [38] give directly $\delta_{34}\left(r^{2}\right)=0.303 \pm 0.016 \mathrm{fm}^{2}$ and $\delta_{24}\left(r^{2}\right)=0.092 \pm 0.005 \mathrm{fm}^{2}$, where the label 4 denotes the ground-state of $\mathrm{Sm}^{148}$. Taking the difference gives

$$
\left.\delta_{32}\left(r^{2}\right)=0.211 \pm 0.017 \mathrm{fm}^{2} \text { ( } 3 \sigma \text { error }\right) .
$$

For the radius of the $\mathrm{Sm}^{150}$ nucleus to insert in (15), we use equations (50) and (51) on page 568 of [36], which give

$$
R_{1}=8.11 \mathrm{fm} .
$$

From (43), (44) and (45), we find

$$
\alpha \frac{d E_{r}}{d \alpha}<-(1.09 \pm 0.09) \mathrm{MeV} .
$$


The estimate (46), obtained here directly from measurements of the small charge-radius difference (44) between $\mathrm{Sm}^{150}$ and $\mathrm{Sm}^{149}$, agrees with the result obtained by differencing the phenomenological Bethe-Weizsäcker formula (droplet model). The latter formula estimates the nuclear Coulomb energy as $\left\langle H_{c}\right\rangle=0.717 Z(Z-1) A^{-1 / 3} \mathrm{MeV}$ [39]. Taking the difference between $\mathrm{Sm}_{62}^{150}$ and $\mathrm{Sm}_{62}^{149}$ (and arguing as above that excited states are less charge concentrated) yields the inequality $\alpha d E_{r} / d \alpha<-1.14 \mathrm{MeV}$, which is compatible with the result (46). By contrast, the Bethe-Weizsäcker formula overestimates by about a factor two the $\alpha$-sensitivity of the resonance energy $E_{r}^{\text {now }}=0.0268 \mathrm{eV}$, for the radiative capture of neutrons by $\mathrm{Gd}_{64}^{155}$. This follows (using the same method as above) from the fact that isotope-shift measurements reported in [40] yield

$$
\left\langle r^{2}\right\rangle_{156}-\left\langle r^{2}\right\rangle_{155}=0.097 \pm 0.005 \mathrm{fm}^{2},
$$

which is less than half the Samarium difference (44). As a consequence the $\alpha$-sensitivity parameter $\left|\alpha d E_{r} / d \alpha\right|$ of the $\mathrm{Gd}^{155}$ resonance is less than half that of the $\mathrm{Sm}^{149}$ resonance.

We are now in position to convert the bound (28) obtained above from our analysis of Oklo data into a bound on the variation of $\alpha$. To be conservative we use the worst $3 \sigma$ limit on the $\alpha$-sensitivity of $E_{r}$ obtainable from (46), namely

$$
\left|\alpha \frac{d E_{r}}{d \alpha}\right|>(1.09-0.09) \mathrm{MeV}=1.0 \mathrm{MeV}
$$

Combining (48) with the bound (28) on the shift $\Delta=E_{r}\left(\alpha^{\text {Oklo }}\right)-E_{r}\left(\alpha^{\text {now }}\right)=$ $-\left|\alpha d E_{r} / d \alpha\right|\left(\alpha^{\text {Oklo }}-\alpha^{\text {now }}\right) / \alpha$ yields our final result

$$
-0.9 \times 10^{-7}<\frac{\alpha^{\text {Oklo }}-\alpha^{\text {now }}}{\alpha}<1.2 \times 10^{-7},
$$

which we consider as a $95 \%$ C.L. limit.

Though we have been very conservative in our analysis, our result (49) confirms the main claim of Refs. [13, 114, 15], namely that Oklo rare-earth data are extremely sensitive probes of a possible variation of $\alpha$ : the surprisingly good $\sim 10^{-7}$ bound comes mainly from the $10^{7}$ amplification factor between the $\mathrm{MeV}$ level in $\alpha d E_{r} / d \alpha$ (which is physically clearly understood from the Bethe-Weizsäcker formula) and the $0.1 \mathrm{eV}$ level of the value of the Samarium resonance (with respect to the threshold). 
Let us note also the consistency of the approximations we made: a change $\delta \alpha / \alpha \sim 10^{-7}$ has a totally negligible effect in all the quantities (such as $\gamma_{n}$ or $\Gamma$ ) depending at most polynomially on $\alpha$, and its effect on the dimensionless ratio $\hat{\sigma}_{149} / \hat{\sigma}_{143}$ is dominated by the Breit-Wigner denominator of $\hat{\sigma}_{149}$, i.e. by the change $\partial E_{r}^{149} / \partial \alpha \delta \alpha$. [The contribution coming from the shift of neutron capture resonances on $\mathrm{Nd}^{143}$ is relatively negligible because, from the approximate $Z(Z-1) A^{-4 / 3}$ dependence expected from the BetheWeizsäcker formula, $\delta E_{r}^{143} \lesssim \delta E_{r}^{149} \sim 0.1 \mathrm{eV}$ which is small compared to the near-threshold resonances in $\mathrm{Nd}^{143}$, specifically the one below the threshold at $E_{r}^{143} \sim-6 \mathrm{eV}$.]

In deriving the bound (49), we have implicitly assumed that, during the Oklo phenomenon, $\alpha$ took some fixed value $\alpha^{\text {Oklo }}$ (possibly different from $\left.\alpha^{\text {now }}\right)$. The situation would be more complicated if, at the time, $\alpha(t)$ were oscillating on a time scale smaller than the duration of Oklo. As $\mathrm{Sm}^{149}$ data depend essentially on the value of $\hat{\sigma}_{149}$ at the end of the fission reaction, we expect that the bound (49) restricts the amplitude of the variation of $\alpha$ in many extended scenarios comprising $\alpha$-oscillations.

Dividing (49) by the age of the Oklo phenomenon, we can convert it into a bound on the time derivative of $\alpha$ averaged over the time span separating us from the end of the Oklo phenomenon. [In scenarios where $\alpha$ varies on the Hubble time scale, this averaged time derivative is nearly equal to the present time derivative.] This conversion introduces a further uncertainty, because the age of Oklo is not determined with precision. The geochronological studies suggest an age around $1.8 \times 10^{9} \mathrm{yr}$ [16], while several studies based on nuclear decay time scales gave $\sim 10 \%$ higher values: for instance, $1.98 \times 10^{9} \mathrm{yr}$ [24], $1.93 \times 10^{9} \mathrm{yr}$ [1], and $2.05 \times 10^{9} \mathrm{yr}$ [42]. To remain conservative in our bounds, we shall use the lower, geochronological value. Dividing Eq. (49) by $1.8 \times 10^{9} \mathrm{yr}$, we get the following conservative (95\% C.L.) limit on the time derivative of $\alpha$ averaged over the time since the Oklo reactor was running

$$
-6.7 \times 10^{-17} \mathrm{yr}^{-1}<\frac{\dot{\alpha}}{\alpha}<5.0 \times 10^{-17} \mathrm{yr}^{-1}
$$

This is weaker than Shlyakhter's estimates (which ranged between $\pm 5 \times$ $10^{-18} \mathrm{yr}^{-1}$ [14] and $\pm 10^{-17} \mathrm{yr}^{-1}$ [13, 15]) but rests on a firmer experimental basis. On the other hand, this is between two and three orders of magnitude stronger than the other constraints on the variability of $\alpha$ (see the Introduction). Thanks to recent advances in atomic clock technology, it is 
conceivable (and desirable) that direct laboratory tests might soon compete with the Oklo bound (50).

We have focussed in this paper on the time variation of the fine-structure constant because one can estimate with some confidence the effect of a change of $\alpha$ on resonance energies. It is more difficult to estimate the effect of a change in the Fermi coupling constant $G_{F}$, or, better, in the dimensionless quantity $\beta=G_{F} m_{p}^{2} c / \hbar^{3} \simeq 1.03 \times 10^{-5}$. The estimates of Ref. 443 for the (Weinberg-Salam) weak-interaction contribution to nuclear ground state energies yield $E_{\text {weak }}^{150}-E_{\text {weak }}^{149} \simeq 5.6 \mathrm{eV}$. If one assumes that this gives an approximate estimate of the difference involving the relevant excited state of $\mathrm{Sm}^{150}$, and that there is no cancellation between the effects of changes in $\alpha$ and $\beta$, one finds from Eq. (28) the approximate bound

$$
\begin{gathered}
\frac{\left|\beta^{\text {Oklo }}-\beta^{\text {now }}\right|}{\beta}<0.02, \\
\left|\frac{\dot{\beta}}{\beta}\right|<10^{-11} \mathrm{yr}^{-1} .
\end{gathered}
$$

This bound is more stringent than the limit $|\dot{\beta} / \beta|<10^{-10} \mathrm{yr}^{-1}$ obtained from the constancy of the $K^{40}$ decay rate [2], and is comparable to the limit derived from Big Bang nucleosynthesis: $\left|\beta^{\mathrm{BBN}}-\beta^{\text {now }}\right| / \beta<0.06$ 44.

Deducing from Oklo data a limit on the time variation of the "strength of the nuclear interaction" poses a greater challenge. First, one must notice that, as remarked in Section 2, only dimensionless ratios of nuclear quantities, such as $\sigma_{149} / \sigma_{143}$, can be extracted from Oklo data. Within the QCD framework, one generally expects any such dimensionless ratio to become a (truly constant) pure number in the "chiral" limit of massless quarks.

Time variation of such a dimensionless ratio is then linked (in QCD) with possible changes in the subleading terms proportional to the mass ratios $m_{q} / m_{p}$, where $m_{q}$ denotes the masses of the light quarks. However, the chiral limit of nuclear binding energies is tricky because of non-analyticity effects in $m_{q}$. The present chiral perturbation technology does not allow one to estimate the dependence of nuclear quantities such as $E_{r}^{149}$ or $\sigma_{149} / \sigma_{143}$ on $m_{q} / m_{p}$. One, however, anticipates that Oklo data might provide a very stringent test (probably at better than the $10^{-7}$ level) on the time variation of $m_{q} / m_{p}$. To separate unambiguously the effects of variations in $\alpha$ and $m_{q} / m_{p}$, it would be necessary to extract from Oklo data several independent 
measured quantities (e.g. by analyzing in detail the effects of resonance shifts in $\mathrm{Gd}^{155}$ and $\left.\mathrm{Gd}^{157}\right)$.

\section{Acknowledgments}

We thank A. Michaudon and B. Pichon for informative communications about nuclear data and M. Ganguli and E. Hansen for helping us to obtain the information relevant to the Oklo phenomenon. The work of T. Damour at the Institute for Advanced Study was supported by the Monell Foundation.

\section{References}

[1] P.A.M. Dirac, Nature 139 (1937) 323; Proc. Roy. Soc. A165 (1938) 199.

[2] F.J. Dyson, The fundamental constants and their time variation in Aspects of Quantum Theory, eds A. Salam and E.P. Wigner (Cambridge University Press, Cambridge, 1972), pp 213-236.

[3] F.J. Dyson, Variation of constants in Current Trends in the Theory of Fields, eds J.E. Lannutti and P.K. Williams (American Institute of Physics, New York, 1978) pp 163-167.

[4] P. Sisterna and H. Vucetich, Phys. Rev. D41 (1990) 1034.

[5] D.A. Varshalovich and A.Y. Potekhin, Space Science Reviews 74 (1995) 259 .

[6] M.B. Green, J.H. Schwarz and E. Witten, Superstring theory (Cambridge University Press, Cambridge, 1987).

[7] T. Damour and A.M. Polyakov, Nucl. Phys. B423 (1994) 532; Gen. Rel. Grav. 26 (1994) 1171.

[8] M.P. Savedoff, Nature 178 (1956) 688.

[9] J.N. Bahcall and M. Schmidt, Phys. Rev. Lett. 19 (1967) 1294.

[10] D.A. Varshalovich, V.E. Panchuk and A.V. Ivanchick, Astronomy Letters 22 (1996) 6. 
[11] L.L. Cowie and A. Songaila, Astrophys. J. 453 (1995) 596.

[12] J.D. Prestage, R.L. Tjoelker and L. Maleki, Phys. Rev. Lett. 74 (1995) 3511.

[13] A.I. Shlyakhter, Nature 264 (1976) 340.

[14] A.I. Shlyakhter, Direct test for the constancy of fundamental nuclear constants using the Oklo natural reactor, Preprint $n^{\circ} 260$, Leningrad Nuclear Physics Institute, Leningrad, September 1976.

[15] A.I. Shlyakhter, Direct test of the time-independence of fundamental nuclear constants using the Oklo natural reactor, ATOMKI Report A/1 (1983).

[16] Le Phénomène d'Oklo (The Oklo Phenomenon) Proceedings of a symposium on the Oklo phenomenon, Libreville, Gabon, June 1975 (International Atomic Energy Agency, Vienna, 1975).

[17] Les Réacteurs de Fission Naturels (Natural Fission Reactors), Proceedings of a meeting on natural fission reactors, Paris, France, December 1977 (International Atomic Energy Agency, Vienna, 1978).

[18] M. Maurette, Ann. Rev. Nuc. Sci 26 (1976) 319.

[19] Y.V. Petrov, Sov. Phys. Usp. 20 (1977) 937.

[20] J.C. Ruffenach et al., in Ref. [16], p. 371.

[21] H. Bassière et al., in Ref. [16], p. 385.

[22] M. Neuilly and R. Naudet, in Ref. [16], p. 541

[23] J.C. Ruffenach et al., Earth and Planetary Science Letters 30 (1976) 94.

[24] J.C. Ruffenach, in Ref. [17], p. 441.

[25] J. Cesario, D. Poupard and R. Naudet, in Ref. [17], p. 473.

[26] F.W. Walker, J.R. Parrington and F. Feiner, Nuclides and Isotopes, Fourteenth Edition (General Electric Company, San Jose, 1989). 
[27] B.F. Rider, Compilation of Fission Product Yields, NEDO-12154-3(C) (Vallecitos Nuclear Center, Pleasanton, California, 1981).

[28] J.F. Dozol and M. Neuilly, in Ref. [16], p. 357.

[29] M. Neuilly, J.F. Dozol and R. Naudet, in Ref. [17], p. 433.

[30] M. Lucas et al., in Ref. [17], p. 407.

[31] P. Holliger, C. Devillers and G. Retali, in Ref. [17], p. 553.

[32] A.M. Weinberg and E.P. Wigner, The Physical Theory of Neutron Chain Reactors (The University of Chicago Press, Chicago, 1958).

[33] R.J. Vidale, in Ref. [17], p. 235.

[34] B. Poty, in Ref. [17], p. 636.

[35] S.F. Mughabghab, Neutron Cross Sections, Vol. 1, Part B (Academic Press, 1984).

[36] E.W. Otten, "Nuclear Radii and Moments of Unstable Nuclei", pp. 517638 in "Treatise on Heavy-Ion Science, Vol. 8", ed. D. Allan Bromley, [Plenum Publishing Corporation, 1989].

[37] L.D. Landau and E.M. Lifshitz, Quantum Mechanics, Nonrelativistic Theory (Pergamon Press, Oxford, 1965).

[38] H. Brand, B. Seibert and A. Steudel, Z. Phys. A 296 (1980) 281.

[39] P. Möller and J.R. Nix, Atomic Data and Nuclear Data Tables, 39 (1988) 213.

[40] S.K. Borisov et al., Soviet Physics JETP, 66 (1987) 882.

[41] C. Devillers and J. Menes, in Ref. [17], p. 495.

[42] A.J. Gancarz, in Ref. [17], p. 513.

[43] M.P. Haugan and C.M. Will, Phys. Rev. Lett. 37 (1976) 1.

[44] R.A. Malaney and G. Mathews, Phys. Rep. 229 (1993) 147; H. Reeves, Rev. Mod. Phys. 66 (1994) 193. 
Table 1: Effective neutron cross sections of $\mathrm{Sm}^{149}$ computed for 15 Oklo samples using published data.

\begin{tabular}{|c|c|c|}
\hline Sample & Reference & $\hat{\sigma}_{149}$ (kbarn) \\
\hline KN50-3548 & "23 & 93 \\
\hline SC36-1408/4 & 24 & 73 \\
\hline SC36-1410/3 & 24 & 73 \\
\hline SC36-1413/3 & 24 & 83 \\
\hline SC36-1418 & 24 & 64 \\
\hline SC39-1383 & 28,29 & 66 \\
\hline SC39-1385 & 28,29 & 69 \\
\hline SC39-1387 & 28, & 36 \\
\hline SC39-1389 & $28, \overline{29}$ & 64 \\
\hline SC39-1390 & 28,29 & 82 \\
\hline SC39-1391 & 28,29 & 82 \\
\hline SC39-1393 & 28,29 & 68 \\
\hline SC35bis-2126 & $228, \overline{29}$ & 57 \\
\hline SC35bis-2130 & 28,29 & 81 \\
\hline SC35bis-2134 & 28,29 & 71 \\
\hline SC52 1472 & [31] & 72 \\
\hline
\end{tabular}

Table 2: Oklo data corresponding to the extreme cross-section results of Eq. (20). Notation as in Eqs. (16), (17).

\begin{tabular}{|l|c|c|c|c|c|c|c|}
\hline Sample & $N_{5} / N_{8}$ & $N_{144}$ & $N_{147}$ & $N_{148}$ & $N_{149}$ & $\tau\left(10^{21} \mathrm{n} / \mathrm{cm}^{2}\right)$ & $\hat{\sigma}_{149}(\mathrm{kbarn})$ \\
\hline SC35bis-2126 & 0.00568 & 0.22 & 53.86 & 2.39 & 0.44 & 0.92 & 57 \\
KN50-3548 & 0.00465 & 0.16 & 52.63 & 6.90 & 0.19 & 1.25 & 93 \\
\hline
\end{tabular}

Figure 1: Variation of the effective neutron capture cross section of $\mathrm{Sm}^{149}$, $\sigma_{149}$, as a function of a possible shift $\Delta=E_{r}^{\text {Oklo }}-E_{r}^{\text {now }}$ in the lowest resonance energy, for several values of the neutron temperature T. $\sigma_{149}, \Delta$ and $T$ (labelling the curves) are measured in kbarn, $\mathrm{eV}$ and degree Celsius, respectively. The two horizontal lines represent a conservative range of values of $\sigma_{149}$ compatible with Oklo data. 